\title{
Model predictive control made accessible to professional automation systems in fermentation technology
}

\author{
Artur Kuprijanov ${ }^{1,2 *}$, Sebastian Schaepe ${ }^{1}$, Rimvydas Simutis ${ }^{2}$, Andreas Lübbert ${ }^{1}$ \\ ${ }^{1}$ Institute for Biochemistry and Biotechnology, Martin-Luther-University Halle-Wittenberg, Kurt-Mothes-Straße 3, 06120 Halle \\ (Saale), Germany. \\ ${ }^{2}$ Process Control Department, Kaunas University of Technology, Studentu g. 48, LT-3028 Kaunas, Lithuania \\ *Corresponding author \\ artur.kuprijanov@biochemtech.uni-halle.de
}

Received: 7 November 2013; accepted: 18 November 2013; published online: 22 November 2013

This paper has no supplementary material.

\begin{abstract}
The objective of this paper is showing how model predictive control can easily be implemented directly into industrial bioreactor automation systems and thus making this control technology accessible to the fed-batch fermentation processes. By means of a practical example it is shown how to keep the biomass concentration exactly on its predefined path taking the substrate feed rate as the only action variable in a bioreactor that is conventionally equipped with standard measurement devices. The model predictive control algorithm uses a very simple general process model the parameters of which are adapted during the cultivation process. Additionally the feed rate profile corresponding to the desired biomass profile is used as a scheduling variable to adapt to changes in the process dynamics and at the same time for safeguarding
\end{abstract}

Keywords: Model predictive control, model adaptation, DCS, E.coli cultivation, recombinant protein production.

\section{Introduction}

Complex processes require complex control systems. Some fermentation processes can be considered examples for processes that require model aided control techniques. In these cases model predictive control is probably the most sophisticated variant.

Model predictive control uses a process model to predict at every sampling point of the measurement variables the near future of the process, across a limited time horizon. For this period it computes possible variants of control settings in order to determine which one would lead to the best controller performance. The best setting is adopted for the next controller setpoint. At the following sampling point the procedure is repeated and the time horizon is shifted ahead by one time increment. This is why the procedure is often referred to as the receding horizon technique. Its advantage is that predictable changes in the process dynamics can be considered before the controller runs into problems.

MPC has already been discussed with reference to various process industries (Maciejowski, 2001; Rossiter, 2003; Qin and Badgwell, 2003; Camacho and Bordons, 2004; Dittmar and Pfeiffer, 2004, Dittmar and Pfeiffer, 2006; Boudreau and McMillan, 2007). The MPC applications for fermentation processes were based on rapid prototyping software and programmed in Matlab (Jenzsch et al., 2006; Aehle et al., 2012). The success of the latter suggested a user friendly implementation of MPC control strategy into industrial distributed control system (DCS, SIMATIC PCS7) in order to make this powerful technique generally available to fermentation processes.

Such a technique is conceptionally elegant but depicts the difficulty that complex systems required complex models and hence the examples reported in literature require a considerable effort in model design and real time evaluation. This is problematic in practical industrial applications where automation systems are employed that are not optimized for the online solutions of complex models. Here only simple universal process models can be used. In order to make them applicable in model predictive controllers, the only way is a continuous parameter adaptation in order to compensate for modeling uncertainties. Furthermore, the search for the optimal controller parameter setting must be simplified. A general nonlinear optimizer is not applicable for two main reasons. First such software cannot be run in automation systems and secondly they would need too much computing power for such systems. Hence, the search for optimal control profiles across the time horizon must be restricted to paths which are truly distinguishable and possible in a given situation. In practice this reduces the number of solutions of the model equation to a handful of trials.

This is the general philosophy of the work reported here, where it is shown how model predictive control was implemented in a standard Siemens SIMATIC PCS7 system and applied to E.coli culture producing recombinant proteins.

\section{Materials and methods}

\subsection{Experimental set up}

All fermentation were performed with E. coli BL21(DE3) pLysS. Offline measurements were performed with time increments of half an hour: Biomass concentrations were estimated from at-line $\mathrm{OD}_{600}$ measurements performed with a spectral photometer (Shimadzu UV-2102PC). Glucose concentrations were quantitatively determined with an YSIGlucose analyzer. In both cases, the measurement values transferred to the automation system were taken as the mean from 3 repetitions. The RMS values of these means were estimated to be less than $3 \%$. 
The cultivations were performed in a Biostat C 10-Lbioreactor (B.Braun Biotech International, Melsungen, Germany) and operated in the fedbatch mode from the beginning. The cells were cultivated in a defined medium. Compositions of the initial medium and the feed solution are described in detail by (Gnoth et al., 2010). Substrate addition rates were controlled gravimetrically using a SIWAREX FTC weighing module and a Mettler Toledo balance. An autosampler Gilson FC203b was used for automatic and timeprecise sampling of off-line measurements. All other details of the experimental set-up were the same as those reported in Kuprijanov et al. (Kuprijanov et al., 2012a).

The cultivation processes were operated under the control of a distributed control system Siemens SIMATIC PCS7 V7.1 using our Bioreactor Control Toolbox (Kuprijanov et al., 2012b). SIMATIC BATCH is used for the formation and configuration for the recipes of fermentation processes.

\subsection{Model used for predictive control}

Most advanced control systems reported in literature use the specific growth rate $\mu$ as the control variable since this is the key physiological variable characterizing the behaviour of the cells, i.e., the very bioreactors in a production process (e.g., Lee et al., 1989; Shioya, 1992; Yoon et al., 1994; Levisauskas et al., 1996; Soons et al., 2006; Wang et al., 2006; Jenzsch, 2006a). From the product manufacturing perspective, however, it is better to control these processes along the corresponding biomass concentration profile as pointed out by Jenzsch et al., (2006b) as this leads to a better batch-to-batch reproducibility.

Goal of the proposed control system is, thus, to control precisely the developed rational biomass set-point profile during the real cultivation by substrate feed manipulation. A general process model was used to predict the process behavior starting from the current time $t$ across a time period $\left[t, t+\Delta t_{h}\right]$. For the usually applied fed-batch process, it was assumed to be sufficient to consider the state variables biomass concentration $X$, substrate concentration $S$ and culture volume $W$ only. The kinetics was dominated by the specific substrate uptake rate and the specific biomass concentration.

$$
\begin{gathered}
\sigma=\sigma_{\text {max }} \cdot \frac{S}{K_{S}+S} \\
\mu=Y_{X S} \cdot \sigma \cdot\left(1-\frac{X}{X_{\text {max }}}\right) \\
\frac{d S}{d t}=-\sigma \cdot X_{\text {mod }}+\frac{F_{c t r l}}{W} \cdot\left(S_{F}-S\right) \\
\frac{d X_{\mathrm{mod}}}{d t}=\mu \cdot X_{\text {mod }}-\frac{F_{c t r l}}{W} \cdot X_{\text {mod }} \\
\frac{d W}{d t}=F_{c t r l}
\end{gathered}
$$

$\sigma_{\max }$ is the maximum specific glucose consumption rate, $K_{S}$ the saturation constant of substrate, $Y_{x s}$ is the growth yield, $X_{\max }$ the biomass concentration that can maximally be achieved. Long experiences with these systems and the noise levels of the available off- and online process data showed that $Y_{x s}$ is an appropriate model parameter that can be used to adapt the model uncertainties to the practically appearing process data.

Under normal operating conditions, these fedbatch processes run under substrate limitation. Then it can be assumed that the substrate fed to the culture is immediately consumed by the cells and the actual $Y_{x s}$ can be estimated by the current amount of cells and the amount of substrate fed to the reactor. The latter is the feed weight cFeed times the substrate concentration $S_{F}$ of the solution fed to the cells

$$
Y_{X S}^{\prime}(t)=\frac{X(t) \cdot W(t)}{c F e e d(t) \cdot S_{F}}
$$

Using the offline data for biomass and substrate from the "golden batch" and the corresponding feed rate and weight profiles, the interval can be estimated in which $Y_{X S}^{\prime}$ is varying across the fermentation process. This is depicted in Fig. 1.

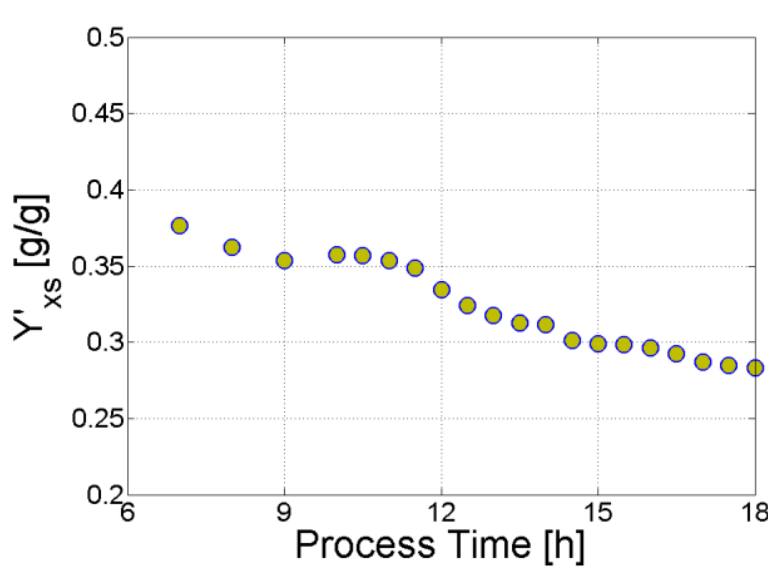

Fig. 1. Time-development of the growth yield $Y^{\prime}$ 'xs during a typical cultivation process controlled with the model predictive controller for study $\mathbf{S 7 4 4}$.

As shown in the Fig. 1, $Y_{x s}^{\prime}$ value varies considerably, showing that such adaptation is necessary during the cultivation.

The initial model parameters were identified using process data from previously performed experiments. For that purpose a standard Nelder-Mead Downhill Simplex technique was used. (here Matlab's fminsearch). The resulting model parameters are compiled in Table.1.

Table 1.

Overview of model parameters used in the MPC control (E. coli bacteria).

\begin{tabular}{|c|c|}
\hline Parameter & Value \\
\hline$K_{s}$ & $0.986 \mathrm{~g} \mathrm{~kg}^{-1}$ \\
\hline$\sigma_{\max }$ & $2.33 \mathrm{~g} \mathrm{~g}^{-1} \mathrm{~h}^{-1}$ \\
\hline$X_{\max }$ & $239.5 \mathrm{~g} \mathrm{~kg}^{-1}$ \\
\hline$Y_{X S}^{\prime}$ & $0.4-0.27 \mathrm{~g} \mathrm{~g}^{-1}$ \\
\hline$S_{F}$ & $600 \mathrm{~g} \mathrm{~kg}^{-1}$ \\
\hline
\end{tabular}

The model adaptation method was tested using simulation studies of the MPC. It was assumed, that the growth yield during the process changes according the profile, observed in real experiments. As shown in the Fig. 2 this adaptation procedure ensured a good controller performance.

The optimal biomass and the corresponding feeding trajectories for the fermentation process can be obtained experimentally using probing control strategy (Åkesson et al., 1999) or using model based approach described by Galvanauskas et al. (Galvanauskas et al., 1998). Very often in practice, however, such a setpoint trajectory for biomass and 
the respective trajectory for the feed rate is taken from an experimentally derived operational procedure that is considered to be desirable, often referred to as the "golden batch".

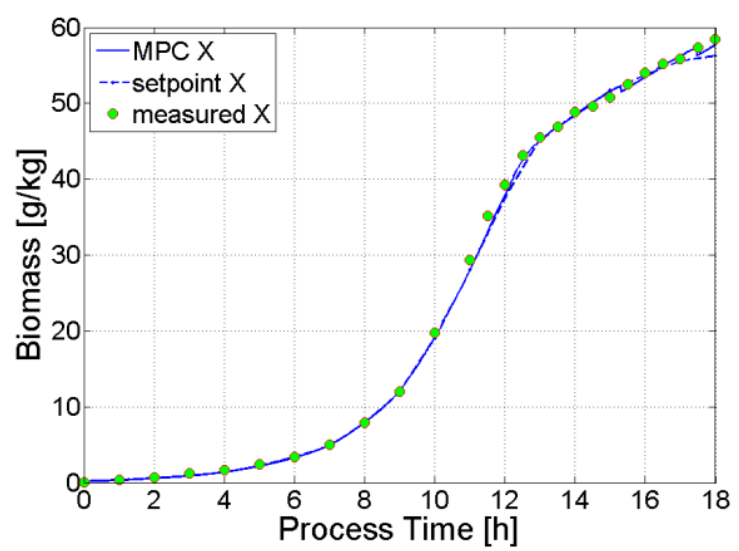

Fig. 2. Biomass measurement profile (symbols) for cultivation $\mathrm{S} 744$ and its set point (dashed) and the simulation (line) of the model predictive controller using the yield adaptation.

\subsection{Keeping the controller simple}

In order to keep the controller as simple as possible, some practical heuristic assumptions were made. The first is that the number of changes of the action variable, the feed rate, that are distinguishable in practice is limited, particularly when the feed rate profile of the undisturbed process is given as a reference $F_{r e f}$ from the golden batch. Hence, an extensive optimization using the standard nonlinear numerical optimization routines is not necessary. Polling across the possible variants is sufficient. Then it is easy to find the best of all realistic variants by means of a simple RMS values.

The integration this process model with MPC algorithm and implementation into real time commercial automation system is an interesting and actual problem.

\section{Implementation of the MPC}

\subsection{Implementation tools}

In the work reported here, SIMATIC STEP7 was used for programming the MPC controller module. This is a specialized programming languages for PLCs and follows the DIN EN 61131-3 standard.

In order to implement the MPC module into PLC, the structured control language (SCL) was taken using an incremental programming approach. SCL is a Pascal-like highlevel language well suited for the implementation of complex algorithms (Berger, 2001).

The continuous function chart (CFC) language was used for logically interconnecting the function blocks, the parameterization of all blocks as well as for the configuration of program sequences. This language is an appropriate instrument to generate the DCS-templates (Pfeiffer, 2007).

As the controller needs two profiles for the controlled variable, in the accompanying example the biomass profile, and the corresponding profile of the scheduling variable, here the substrate feed rate profile of the golden batch, an appropriate tool is required that takes over these data records and make them available to the controller module.

For that purpose a trajectory loading faceplate is designed, which allows to write up to 8000 setpoint values (reading cycles) for biomass setpoint trajectory and for predefined feeding trajectory. This module writes the data directly into the CPU's global data block (DB). The MPC function block can then easily access these trajectories any time. In this way time consuming communications between the MPC and SCADAworkstation, where such trajectories are usually stored, were avoided (Fig. 3). In case of any short blackout of the operator station, these profiles are still available and there will be no impact on the controller performance.

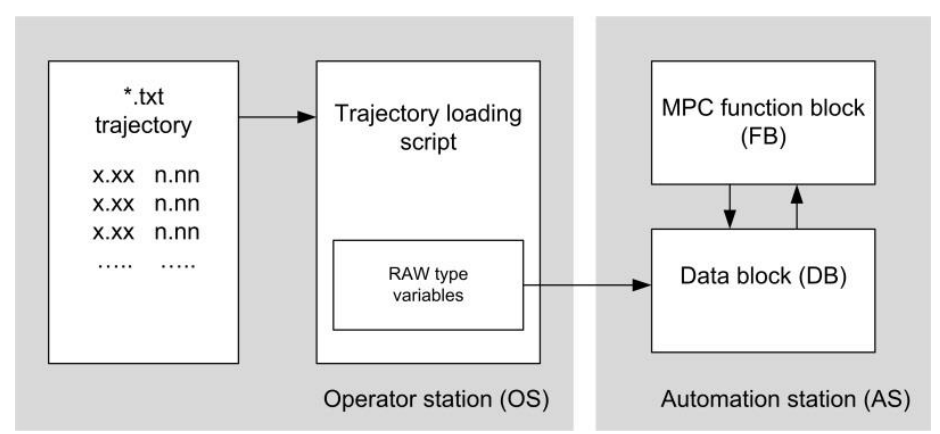

Fig. 3. The picture represents a structure of saving biomass setpoint and predefined feeding trajectories directly into PLC memory.

\subsection{MPC algorithm and configuration}

The MPC function block for fermentation processes has four main functions:

- managing the offline measured data;

- model parameter adaptation;

- $\quad$ solving the model equation;

- $\quad$ solving the optimization problem.

The algorithmic representation of program functionality is shown in Fig. 4.

\subsubsection{Managing the offline measured data}

An important characteristic of current fermentation processes is that many key variables such as biomass and substrate concentrations are measured offline. These data are usually available 15-30 minutes after sampling and the measurement values are then made available to the running fermentation. As this procedure is usually not available in currently used automation systems. Then it becomes important supporting this sampling event as well as the uptake of the measurement values.

The offline measurement procedure starts with a registration of the actual sampling time. This instant of time is stored in the controllers instance data block (IDB) where simultaneously space is allocated for the offline measurement data to be filled in at a later point in time. The program also synchronizes this with the intern cycle at which the current online measured data (culture weight and total glucose-feed weight) are stored (also in the IDB).

The reservation procedure can be triggered automatically by an automatic sampling device or manually by the laboratory personnel using a special "New sample" button. When the offline measurement values are available, within 15-30 min after sampling, they can be made available to the MPC module either via the keyboard or directly from the LIMS system installed in the laboratory. The corresponding MPC visualisation faceplate is shown in Fig. 5. 


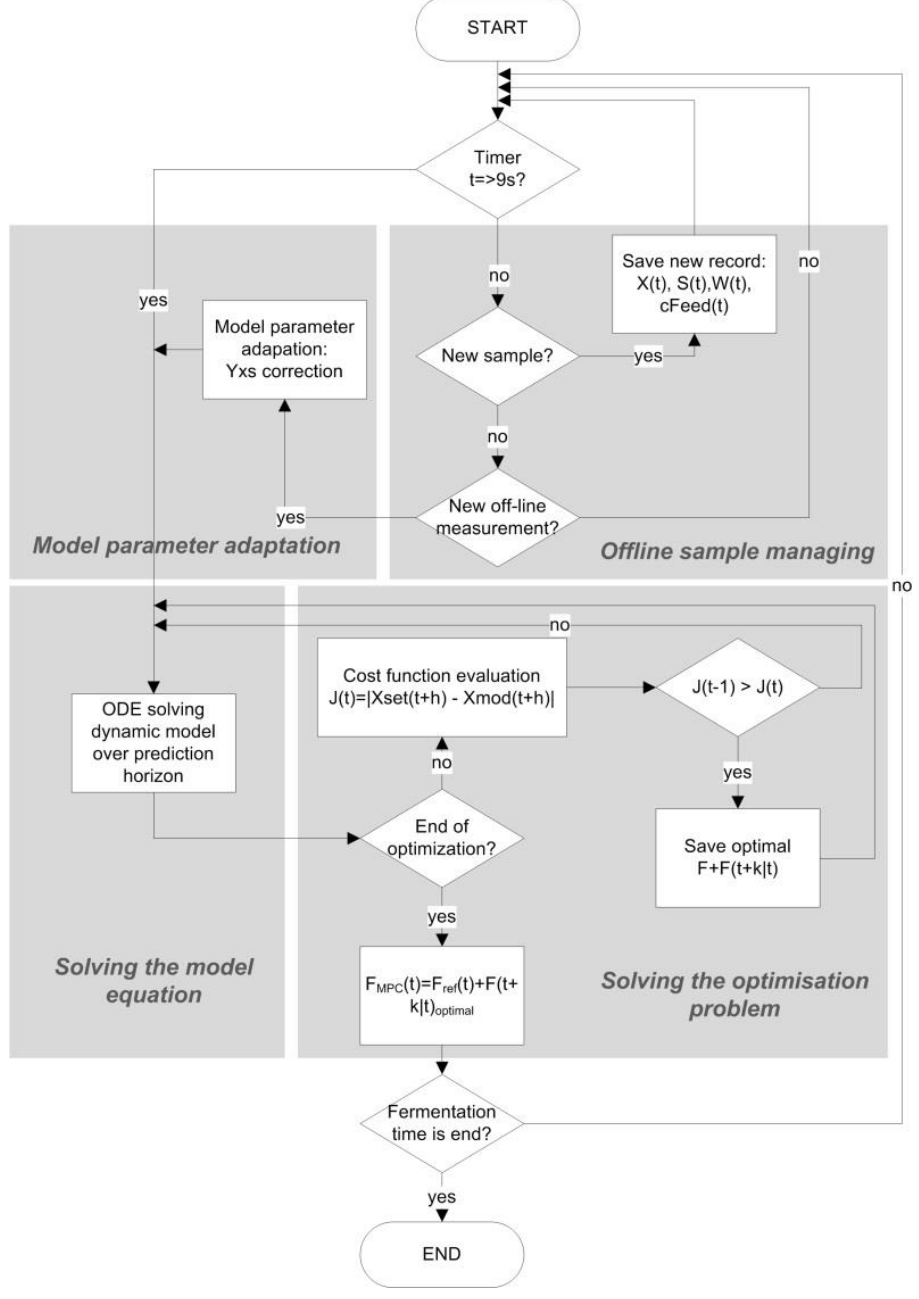

Fig. 4. Program algorithm of MPC programmed in the function block. The general procedure of iterative search of optimal feeding trajectory.

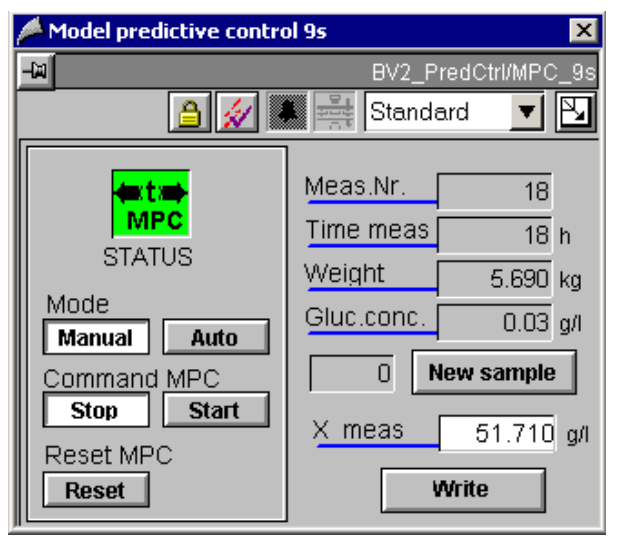

Fig. 5. Visualisation of the MPC controller in the Simatic PCS7 system. With this faceplate the operator can manually set a time stamp and automatically allocates space for the off-line data to be expected from the sample upon its analysis. When these data are available, the faceplate allows writing them into that memory elements.

The new offline measurement values are immediately utilized by the running MPC controller.

\subsubsection{Model parameter adaptation}

As explained above the actual biomass yield on substrate $Y_{x s}^{\prime}$ is estimated from the expression

$$
Y_{X S}^{\prime}\left(t_{s}\right)=\frac{X\left(t_{s}\right) \cdot W\left(t_{s}\right)}{c F e e d\left(t_{s}\right) \cdot S_{F}} \text { and } \mu=Y_{X S}^{\prime} \cdot \sigma
$$

When the controller is running the estimate is based on offline measurement data at the sample time $t_{s}$ for $X$ and $S$ as well as the current online values of reactor's weight $W$ and the glucose feed weight $c$ Feed.

\subsubsection{Solving the model equation and determining the controller action}

Predicting the process behaviour across the time horizon requires numerically solving the model equations. For that purpose a simple Euler algorithm was chosen with the time increments ( $9[\mathrm{~s}])$ of the feed rate profile of the "golden batch" deposited in the controller module as described above. This method was chosen because of its simplicity and thus its easy implementation. It proved to be sufficiently accurate.

Basis of the controller action is the reference feed rate profile $F_{\text {ref }}(t)$ stored in the data block of the PLC as described before. The MPC controller determines an optimal correction $\Delta F$ to this basic profile across the time horizon

$F_{M P C}(t+k \mid t)=F_{r e f}(t+k \mid t) \pm \Delta F(t+k \mid t) \quad k=0,1, \ldots n_{h}(3.2)$

For various choices of $\Delta F$, these feed rate segments are used to compute process responses for chosen prediction horizon. As the feed pumps do not allow for infinitesimal changes of $F_{M P C}$, only 30 versions of $\Delta F$ within $\pm 10 \%$ around the reference feed rate profile were computed.

The range $\Delta F$ depends on the accuracy and robustness of the reference profile $F_{\text {ref }}$ as well as of the expected deviations from that profile. Clearly, when the deviations are larger than $10 \%$ the controller can only correct for that when its capture domain is larger. The value of $10 \%$ was determined from numerical simulation experiments.

Since jumps in the manipulated variable can cause disturbances in other critical process variables, for instance in the dissolved oxygen concentration in the bioreactor, they were avoided by taking an exponential correction approach for $\Delta F$

$F_{M P C}(t+k \mid t)=F_{r e f}(t+k \mid t) \mp\left(1-\exp \left(\mu_{s e t} \cdot(t+k \mid t)\right) \quad k=0,1, \ldots N_{h}(3.3)\right.$

Here the $\mu_{\text {set }}$ values can be changed stepwise in $\left[0 \ldots \mu_{\max }\right]$ range. As a constraint to $F_{M P C}$, the pump characteristics were taken

$$
F_{r e f}(t+k \mid t)-\Delta F_{\min } \leq F_{M P C}(t+k \mid t) \leq F_{r e f}(t+k \mid t)+\Delta F_{\max } \quad k=0,1, \ldots N_{h}(3.4)
$$

As with all other advanced controllers, the MPC is started after a fermentation time of $5[\mathrm{~h}]$ only, since up to that time instant the data from the process are usually not reliable enough as to leading to a better process performance than an open loop controller along an exponential growth profile with $\mu<\mu_{\max }$ (Jenzsch et al., 2006, Jenzsch et al., 2007). In this phase the reference trajectory $F_{r e f}$ is taken to control the process.

The MPC controller is activated upon the first offline sampling instance $t_{s}>5[\mathrm{~h}]$. The adaptation of $Y_{x s}$ parameter and following corrective prediction is carried out when the offline measurement values $X\left(t_{s}\right)$ and $S\left(t_{s}\right)$ are available within the automation station. The horizon for this corrective prediction is 
$\left[t_{s}, t_{h}\right]$, this include the feed rate profile up to current time instant $\left[t_{s}, t\right]$. For the rest of the time horizon 30 different variants for $\Delta F\left(t, t_{h}\right)$ were taken. From the variants computed with these feed rate profiles, the one with the least deviation between the corresponding biomass and its setpoint profile segment is taken for control. Even from this profile only the feed value for the current time $t$ is sent to the feeding valve. Upon each such cycle, the time horizon is then shifted $9[\mathrm{~s}]$ ahead and the procedure is repeated for time horizon $\left[t, t_{h}\right]$. For that new cycle the initial values for the predictions were taken from the last optimal $X$ biomass trajectory. The additional information in this cycle comes from the shifted biomass setpoint profile within the new time horizon. This will lead to a slightly different optimal feeding profile within that time interval $t \leq t_{h}$.

The duration of the receding time horizon was chosen to $1[\mathrm{~h}]$. This value was derived from simulation studies made beforehand and was compared experimentally with other variants $[0 . .2 \mathrm{~h}]$. An adaptation of the model was also made hourly. Within the last hour, the time horizon shrinks until its time span is zero at $18[\mathrm{~h}]$ fermentation time. Finally, the time steps for the controller cycle was chose to be the same as the time increment for storing the online measurements.

If an unexpected disturbance occurs during the cultivation process, there is a possibility to switch MPC controller into the safe open-loop operation mode. In that case the feeding reference trajectory $F_{r e f}$ will be taken to finish the fermentation process. In the case of an abnormal or missing offline measurement the controller doesn't activate the model adaptation function and waits for the next automatic or manual sampling action.

\section{Results and discussion}

The proposed model predictive control procedure was implemented into our BioPAT library and run in our Siemens SIMATIC PCS7 automation system to control E.coli culture.

The controller is able to keep the process well within its specification limits. From the manufacturing perspective, however, the controller performance is importantly characterized by the batch-to-batch reproducibility that can be assured with such a controller and which clearly sets out the process quality. This way of performance quantification can best be appraised by plotting several MPC-controlled fermentation runs into a common plot. Fig. 6 shows 5 such validation runs. Obviously the controller works perfectly over the entire fermentation process control time. The absolute deviations shown in the lower part of the plot clearly demonstrate the very good batch-to-batch reproducibility obtained with the MPC.

The upper graph (Fig. 6) shows the data for the controlled variable, the lower one the absolute deviations of the validation runs from the set points. The symbols are the same in both plots. An important point to note here is that this process performance could be obtained with a minimum of measurement expenses. Comparably good batch-to-batch reproducibility was previously only obtained by controlling the total mass of carbon dioxide produced (tcCPR-Control), e.g, Jenzsch et al. (Jenzsch et al., 2007). That approach required measurements of the carbon dioxide volume fraction in the vent line of the fermenter and thus an offgas analysis that is not generally available at production fermenters. For the MPC controller, only the offline measurements of biomass via OD measurements and substrate concentrations measurements via (glucose analyzers) are needed. These measurements are being performed at most production reactors. The only online measurements that are used are feed rate and culture weight. While the former is generally measured, the balance needed to measure the second one is sometimes not directly available and then needs to be estimated from the added mass of substrate and the initial culture weight.
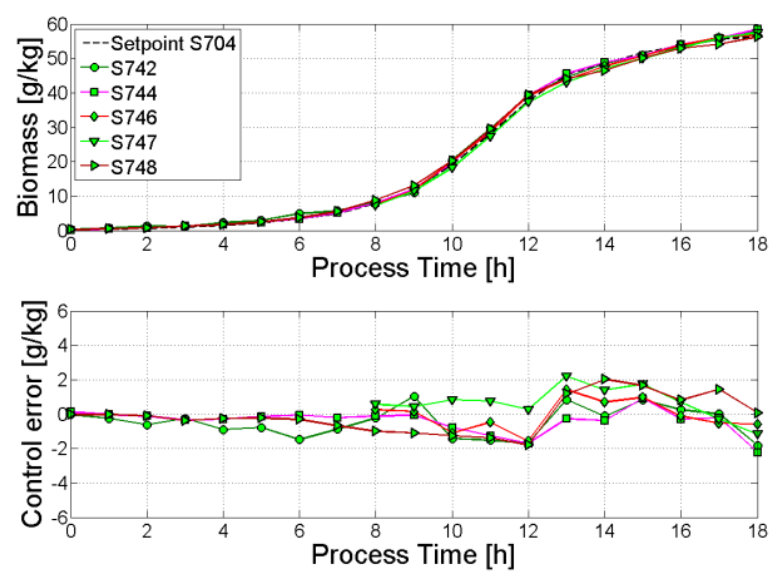

Fig. 6. Results of 5 validation runs for the proposed MPC controller together with the set point profile which was taken from a previously optimized fermentation (Study S704).

\section{Conclusion}

In conclusion, the most important results of the work behind this report are:

- A simple but powerful version of an adaptive model predictive controller was developed and tested in many real fermentations on the laboratory scale.

- The corresponding software was developed in form of a module for the generally applicable professional automation system Siemens SIMATIC PCS7 and is thus portable (including its faceplates) to other cases of applications of this software.

- The implementation of the model predictive controller was constructed without utilizing external general purpose computing elements. The algorithms were directly implemented in the automations system thus avoiding complex synchronization expenses for external software.

- An appropriate way of handling offline measurement data together with the corresponding online measurements was an essential hurdle during the development. This was solved in a simple way.

- A priori information about changes in the process dynamics could be extracted from the substrate feeding profiles of the "golden batch" from which the rational biomass setpoint profile of the process was taken.

\section{Acknowledgements}

This research was supported by the Research Council of Lithuania (grant No. MIP-056/2013) and Siemens AG, Karlsruhe, Germany. Their help is gratefully acknowledged. 


$\begin{array}{ll}\text { Abbreviation List } \\ \text { MPC } & \text { Model Predictive Control } \\ \text { DCS } & \text { Distributed Control System } \\ \text { SCL } & \text { Structured Control Language } \\ \text { CFC } & \text { Continuous Function Chart } \\ \text { DB } & \text { Data Block } \\ \text { IDB } & \text { Instance Data Block } \\ \text { SCADA } & \text { Supervisory Control and Data Acquisition } \\ \text { LIMS } & \text { Labour Information's Management System } \\ \text { RMS } & \text { Route Mean Square } \\ \text { tcCPR } & \text { Total Cumulative Carbon Production Rate }\end{array}$

\section{References}

Aehle, M., Bork, K., Schaepe, S., Kuprijanov, A., Hostkorte, R., Simutis, R., Lübbert, A. (2012). "Increasing batch-to-batch reproducibility of CHOcell cultures using a model predictive control approach" Cytotechnology, vol. 64, pp. 623-634. http://dx.doi.org/10.1007/s10616-012-9438-1

Åkesson, M., Hagander, P., Axelsson, J.P. (1999). "A probing feeding strategy for Escherichia coli cultures" Biotechnology Techniques, vol. 13, iss.8, pp. 523-528. http://dx.doi.org/10.1023/A:1008906517033

Berger, H. (2001). Automating with STEP 7 in STL and SCL: SIMATIC S7300/400 Programmable Controllers, Wiley.

Boudreau, M. A. and McMillan, G. K. (2007). New Directions in Bioprocess Modeling and Control: Maximizing Process Analytical Technology Benefits, ISA.

Camacho, E.F., Bordons, C. (2004). Model predictive control in the process industry, Springer, London.

Dittmar, R., Pfeiffer, B.M (2004). Modellbasierte prädiktive Regelung: eine Einführung für Ingenieure, Oldenbourg. http://dx.doi.org/10.1524/9783486594911

Dittmar, R., Pfeiffer, B.-M. (2006). "Modelbasierte prädiktive Regelung in der industriellen Praxis" Automatisierungstechnik, Oldenbourg Wissenschaftsverlag, vol. 54, iss. 12, pp. 590-601. http://dx.doi.org/10.1524/auto.2006.54.12.590

Galvanauskas, V., Simutis, R., Volk, N., Lübbert, A. (1998). "Model based design of a biochemical cultivation process" Bioprocess Engineering, vol. 18 , iss. 3, pp. 227-234. http://dx.doi.org/10.1007/s004490050435

Gnoth, S., Simutis, R., Lübbert, A. (2010). "Selective expression of the soluble product fraction in Escherichia coli cultures employed in recombinant protein production processes" Applied Microbiology and Biotechnology, vol. 87, iss. 6, pp. 2047-2058. http://dx.doi.org/10.1007/s00253-010-2608-1

Jenzsch, M., Gnoth, S., Kleinschmidt, M., Simutis, R. Lübbert, A. (2007). "Improving the batch-to-batch reproducibility of microbial cultures during recombinant protein production by regulation of the total carbon dioxide production" Journal of Biotechnology, vol. 128(4), pp. 858-867. http://dx.doi.org/10.1016/j.jbiotec.2006.12.022

Jenzsch, M., Gnoth, S., Kleinschmidt, M., Simutis, R., Lübbert, A. (2006). "Improving the batch-to-batch reproducibility in microbial cultures during recombinant protein production by guiding the process along a predefined total biomass profile" Bioprocess and Biosystems Engineering, vol. 29(5 6), pp. 315-321. http://dx.doi.org/10.1007/s00449-006-0080-1

Jenzsch, M., Simutis, R., Lübbert, A. (2006). "Generic model control of the specific growth rate in E.coli cultivations" Journal of Biotechnology, vol. 122, iss. 4, pp. 483-493. http://dx.doi.org/10.1016/j.jbiotec.2005.09.013

Kuprijanov, A., Schaepe, S., Aehle, M., Simutis, R., Lübbert, A. (2012a). "Improving cultivation processes for recombinant protein production" $\mathrm{Bi}$ oprocess and Biosystems Engineering, Springer Berlin, vol. 35(3), pp. 333-340. http://dx.doi.org/10.1007/s00449-011-0571-6

Kuprijanov, A., Schaepe, S., Aehle, M., Simutis, R., Lübbert, A. (2012b) "Toolbox for Automatic Control of the Fermentation Processes for the Production of Recombinant Therapeutic Protein" Electrical and Control Technologies - 2012, Kaunas University of Technology, IFAC Committee of National Lithuanian Organisation, Lithuanian Electricity Association, Full Member of EUROELECTRIC. Kaunas: Technologija. ISSN $1822-5934$

Lee, J., Youn-Hee, C., Shin-Kwon, K., Hyung-Hwan, P., Ik-Boo, K. (1989). "Production of human leukocyte interferon in Escherichia coli by control of growth rate in fed-batch fermentation" Biotechnology Letters, vol. 11(10), pp. 695-698. http://dx.doi.org/10.1007/BF01044099

Levisauskas, D., Simutis, R., Borvitz, D., Lübbert, A. (1996). "Automatic control of the specific growth rate in fed-batch cultivations processes based on exhaust gas analysis" Bioprocess Engineering, vol. 15(3), pp.145-150. http://dx.doi.org/10.1007/BF00369618

Maciejowski, J. (2001). Predictive control with constraints, Prentice Hall.

Pfeiffer, B-M. (2007). "Standardisierung gehobener Regelungsfunktionen als Messstellen-Typen" GMA-Kongress 2007, Baden-Baden. VDI-Berichte Nr. 1980, VDI-Verlag, Düsseldorf, pp. 83-94

Qin S. J. and Badgwell T. (2000). "An Overview of Nonlinear Model Predictive Control Applications". Allgöwer F. and Zheng A. Nonlinear Model Predictive Control, Birkhäuser Basel. Part III, vol. 26, pp. 369-392. http://dx.doi.org/10.1007/978-3-0348-8407-5_21

Qin, S.J., Badgwell, T.A. (2003). "A survey of industrial model predictive control technology" Control Engineering Practice, vol. 11, iss. 7, pp. 733-746. http://dx.doi.org/10.1016/S0967-0661(02)00186-7

Rossiter, J.A. (2003). Model predictive control: A practical approach, CRC Press LLC, Boca Rotaton.

Shioya, S. (1992). "Optimization and control in fed-batch bioreactors" Modern Biochemical Engineering, Springer Berlin Heidelberg, vol. 46, pp. 111 142. http://dx.doi.org/10.1007/BFb0000708

Soons, Z.I.T.A., Voogt, J.A., van Straten, G., van Boxtel, A.J.B. (2006). "Constant specific growth rate in fed-batch cultivation of Bordetella pertussis using adaptive control" Journal of Biotechnology, 125, 252-268. http://dx.doi.org/10.1016/j.jbiotec.2006.03.005

Wang, F., Du, D., Li, Y., Chen, J. (2006). "Regulation of CCR in the CGTase production from Bacillus macorous by the specific cell growth rate control" Enzyme and Microbial Technology.vol. 39(6), pp. 1279-1285. http://dx.doi.org/10.1016/j.enzmictec.2006.03.014

Yoon, S.K., Kang, W.K., Park, T.H. (1994). "Fed-batch operation of recombinant Escherichia coli containing trp promoter with controlled specific growth rate" Biotechnology and bioengineering, vol. 43(10), pp. 995-999. http://dx.doi.org/10.1002/bit.260431013 